\title{
Energy of a pointlike neutron in an external electromagnetic field
}

\author{
Jesus Saenz®* \\ Institute of Engineering and Technology, Universidad Autónoma de Ciudad Juárez, 32310 Ciudad Juárez, \\ Mexico and Department of Physics, New Mexico State University, Las Cruces, New Mexico 88003, USA \\ Michael Engelhardt $\oplus^{\dagger}$ \\ Department of Physics, New Mexico State University, Las Cruces, New Mexico 88003, USA \\ Roman Höllwieser@ ${ }^{\ddagger}$ \\ Department of Physics, University of Wuppertal, 42119 Wuppertal, Germany \\ and Department of Physics, New Mexico State University, Las Cruces, New Mexico 88003, USA
}

(Received 4 September 2020; revised 21 June 2021; accepted 3 August 2021; published 3 September 2021)

\begin{abstract}
A pointlike neutron in an external electromagnetic field experiences a shift in energy that mimicks the effect of an actual structural deformation of an extended neutron, i.e., a proper polarizability. In order to be able to differentiate between the former and the latter, a Foldy-Wouthuysen transformation is constructed which yields the energy shift of a pointlike neutron quadratic in the external field in a derivative expansion, generalizing a long-known result for the dipole electric polarizability due to Foldy. The ten leading Foldy contributions to the energy are determined for a zero-momentum neutron. In addition, eliminating the momentum operator in favor of the velocity operator, analogous results are derived for a zero-velocity neutron. In this case, operator ordering ambiguities are encountered that permit only a determination of eight of the ten Foldy terms.
\end{abstract}

DOI: 10.1103/PhysRevD.104.056002

\section{INTRODUCTION}

Electromagnetic fields polarize nucleons by coupling to the electric charges of their quark constituents. The degree to which nucleons are susceptible to these polarization effects constitutes a basic question about nucleon structure. For sufficiently weak fields, such effects are quantified through polarizabilities, which characterize the linear response of the nucleon to the electromagnetic field; in terms of an effective Hamiltonian, polarizabilities are coefficients of terms quadratic in the electromagnetic field.

In addition, the fields can be classified according to their space-time variation, starting with the most basic case of constant electric and magnetic fields that induce the socalled dipole polarizabilities; generalizing to space-time dependent fields, the effective Hamiltonian can then be organized into a derivative expansion. The leading polarizability-related terms of the effective Hamiltonian in the

*jmsaenzv@nmsu.edu

engel@nmsu.edu

thoellwieser@uni-wuppertal.de

Published by the American Physical Society under the terms of the Creative Commons Attribution 4.0 International license. Further distribution of this work must maintain attribution to the author(s) and the published article's title, journal citation, and DOI. Funded by SCOAP ${ }^{3}$. expansion in space-time derivatives of the electromagnetic field $\operatorname{read}^{1}[1]$

$$
\begin{aligned}
H_{\mathrm{eff}}= & -\frac{1}{2}\left[\alpha_{E} E^{2}+\beta_{M} B^{2}+\gamma_{E 1} \sigma \cdot(E \times \dot{E})+\gamma_{M 1} \sigma \cdot(B \times \dot{B})\right. \\
& -2 \gamma_{E 2} E_{i j} \sigma_{i} B_{j}+2 \gamma_{M 2} B_{i j} \sigma_{i} E_{j}+\alpha_{E \nu} \dot{E}^{2}+\beta_{M \nu} \dot{B}^{2} \\
& \left.+\frac{1}{6} \alpha_{E 2} E_{i j}^{2}+\frac{1}{6} \beta_{M 2} B_{i j}^{2}+\cdots\right]
\end{aligned}
$$

with the quadrupole strengths of the electric and magnetic fields,

$E_{i j}=\frac{1}{2}\left(\nabla_{i} E_{j}+\nabla_{j} E_{i}\right), \quad B_{i j}=\frac{1}{2}\left(\nabla_{i} B_{j}+\nabla_{j} B_{i}\right)$.

Here, $\alpha_{E}$ and $\beta_{M}$ are the aforementioned dipole electric and magnetic polarizabilities; further, $\gamma_{E 1}, \gamma_{M 1}, \gamma_{E 2}$, and $\gamma_{M 2}$ are the spin polarizabilities, $\alpha_{E \nu}$ and $\beta_{M \nu}$ are the dispersion polarizabilities, and $\alpha_{E 2}$ and $\beta_{M 2}$ are the quadrupole polarizabilities.

It should be noted that casting the response of nucleons to external fields in the local, truncated form (1) constitutes a

\footnotetext{
${ }^{1}$ At variance with [1], the present work employs Gaussian units.
} 
significant assumption. In general, the energy of the nucleon in the presence of external fields is a nonlocal functional incorporating information about space-time correlations between the nucleon wave function and the external field. Adopting the form (1) implies that the nucleon wave function is sufficiently localized such that the nucleon's energy only depends on the local values of the electromagnetic field and its first derivatives; in (1), those quantities are Taylor coefficients of an expansion of the external field around the position of the nucleon, and as such are not themselves functions of space and time anymore, for a given nucleon position. On the other hand, the assumption of a localized nucleon state quantum mechanically clashes with the notion of studying the nucleon at rest, which is generically associated with a spatially extended wave function. The consequent limitations on the applicability of the form (1) will constitute an important aspect of the present study; extended comments follow further below.

As laid out in detail in [1], the ten polarizabilities in (1) can be connected to the amplitudes for nucleon Compton scattering in the low-energy limit. Continued progress in the phenomenological extraction of electromagnetic polarizabilities, which more recently has begun to encompass not only the dipole polarizabilities, but also spin polarizabilities, has been reported in [2-23]. Sensitivity to orders beyond the ones displayed in (1) was considered in $[7,14]$. On the other hand, efforts have been undertaken to evaluate polarizabilities in lattice QCD, by computing hadron mass shifts in the presence of external electromagnetic fields [24-45]. Chiral effective theory serves to connect such lattice data, which are obtained at heavier-than-physical pion masses on finite volumes, to the physical limit $[4,44,46-48]$. In the case of magnetic fields, care must be taken to disentangle the mass shift from the Landau level structure [40-45,49], and effects beyond linear response may contaminate the analysis [50,51]. The subtleties involved in matching the background field calculations performed in lattice QCD to the effective field theory description of scattering amplitudes were examined in detail in $[52,53]$.

The interpretation of hadron mass shifts in terms of polarizabilities is not bereft of subtlety. Already before the advent of the current understanding of nucleon structure in terms of underlying quark and gluon degrees of freedom, it was noted by Foldy [54] that even a pointlike neutron in the presence of a constant electric field experiences an energy shift quadratic in the electric field. The argument can be made quite succinctly: Writing the Dirac equation for a neutral point particle with an (entirely anomalous) magnetic moment $\mu$ as

$$
\left[i \gamma^{\mu} \partial_{\mu}-\frac{\mu}{2} \sigma_{\mu \nu} F^{\mu \nu}-m\right] \psi=0,
$$

the corresponding Dirac Hamiltonian reads [cf. Eq. (26) below for the Dirac structure conventions employed in this work]

$$
H=\alpha \cdot p+i \mu \gamma \cdot E-\mu \Pi \cdot B+\beta m .
$$

If the external field is purely electric and constant, $[H, p]=0$; then, the energy of a zero-momentum neutron, $W^{p=0}$, can be extracted by noting that, for $p=0$, one has $H^{2}=m^{2}+\mu^{2} E^{2}$, and hence

$$
W^{p=0}=\sqrt{m^{2}+\mu^{2} E^{2}}=m+\frac{\mu^{2} E^{2}}{2 m}+\cdots .
$$

The energy shift quadratic in the electric field $E$ mimics the effect of a polarizability, cf. (1). In effect, $\alpha_{E}^{\text {Foldy }}=-\mu^{2} / m$. However, it is not due to an actual structural deformation of the neutron; the neutron was treated as a point particle (at most, one may argue that the anomalous magnetic moment $\mu$ is chiefly a consequence of the neutron's substructure). It may therefore be useful to separate this effect from the effect due to an actual polarization of the neutron $[31,55]$, i.e., subtract the term proportional to $E^{2}$ on the right-hand side of (5) from the mass shift of the neutron obtained in a constant external electric field, in order to extract the dipole electric polarizability proper. Correspondingly, standard phenomenological analyses apportion this contribution to the Born, nonstructure parts of the amplitudes describing nucleon Compton scattering $[8,48]$.

A constant magnetic field $B$, for which solutions of the Dirac equation for particles with and without electric charge and with anomalous magnetic moment are discussed in detail in [56], cf. also [57], does not induce a Foldy-type term analogous to the electric one in (5). Solutions of the Dirac equation for neutral particles in more general forms of magnetic field are discussed in [58], without, however, allowing for a direct identification of Foldy-type coefficients.

Motivated by the advent of experimental data allowing one to extract spin polarizabilities [12,20-22], the purpose of the present work is to expand the treatment of Foldy-type effects for a neutron from the simple dipole polarizability cases highlighted above to all ten polarizabilities defined in Eq. (1). This is achieved by way of constructing an appropriate Foldy-Wouthuysen transformation [59]. An additional aspect that will be taken into account is the one stressed in $[55,60]$, namely, that in the presence of electromagnetic fields, zero momentum and zero velocity are not synonymous. The Foldy contributions to the energy of a neutron in both types of states will be considered. In the case of a zero-velocity neutron, obstructions will be encountered that ultimately only allow one to determine eight of the ten Foldy-type coefficients. These obstructions appear to be symptoms of the general limitation of the definition (1) already noted further above, namely, that the form (1) implies a localization of the neutron wave function that clashes with the notion of studying the neutron at rest, be it in the sense of zero momentum or zero velocity. 
Further expanding upon this latter point, in extracting a local result of the form (1) from an initial description of the neutron in terms of a Dirac Hamiltonian, cf. (4), with external fields depending on space and time, one must take care when invoking the localization assumption. At first sight, a neutron state can certainly become localized despite its momentum being limited to negligible magnitudes as long as it is sufficiently heavy. As one takes the local limit, corrections due to the residual extent of the neutron wave function will vanish as an inverse power of the neutron mass. However, also the Foldy terms at issue here vanish as an inverse power of the neutron mass. Therefore, it is necessary to compare the behavior of the two effects carefully as the local limit is taken. This is explored in Sec. III B and, indeed, the two effects are of the same order. Therefore, the aforementioned corrections due to the residual extent of the neutron wave function must be taken into account in any comprehensive analysis of a concrete physical setting, such as, say, a lattice QCD calculation of the neutron energy (along with, of course, the many other systematic effects arising in such a setting). Nevertheless, it should be emphasized that these corrections depend on further details of the environment in which the neutron is placed, such as boundary conditions, and thus do not constitute intrinsic electromagnetic properties of the neutron on the same footing as the Foldy contributions.

As indicated by this preliminary discussion, the emphasis of the present study lies as much on ascertaining the boundaries of a description in terms of a local effective Hamiltonian of the form (1) as it does on extracting concrete results for the Foldy contributions associated with the polarizabilities in (1) to the extent possible. These limitations will become apparent in more than one aspect, and to exhibit them is as much a goal of this investigation as is the determination of those Foldy-type effects that are accessible in a such a framework.

\section{FOLDY-WOUTHUYSEN TRANSFORMATION}

\section{A. General form of the transformation}

The Foldy-Wouthuysen transformation $[59,61]$ serves to decouple the dynamics of the particle and antiparticle components of a Dirac spinor, at least to a given order in an expansion scheme. Expansion in the inverse particle mass, $1 / m$, yields the relativistic corrections to the nonrelativistic Hamiltonian; here, the expansion parameters will instead be the external electromagnetic field strengths along with their derivatives. To achieve this expansion, the following treatment will largely follow the scheme laid out in [61]. Consider a Hamiltonian of the form

$$
H=\beta m+\mathcal{E}+\mathcal{O},
$$

with even and odd operators characterized by $\beta \mathcal{E}=\mathcal{E} \beta$, $\beta \mathcal{O}=-\mathcal{O} \beta$, inducing an equation of motion

$$
\left(-i \frac{\partial}{\partial t}+H\right) \psi=0
$$

The goal is to transform this (for the purpose of mitigating the effects of $\mathcal{O}$ ) as

$$
U^{\dagger}\left(-i \frac{\partial}{\partial t}+H\right) U \psi^{\prime}=0
$$

where $\psi^{\prime}=U^{\dagger} \psi$. The new Hamiltonian can now be extracted by observing that

$$
\left[-i \frac{\partial}{\partial t}+U^{\dagger}\left(-i \frac{\partial U}{\partial t}\right)+U^{\dagger} H U\right] \psi^{\prime}=0,
$$

i.e., one has the new Hamiltonian

$$
H^{\prime}=U^{\dagger}\left(-i \frac{\partial U}{\partial t}\right)+U^{\dagger}(\beta m+\mathcal{O}) U+U^{\dagger} \mathcal{E} U
$$

In [61], the following transformation is constructed:

$$
U=\frac{\epsilon+m-\beta \mathcal{O}}{\sqrt{2 \epsilon(\epsilon+m)}}
$$

where $\epsilon=\sqrt{m^{2}+\mathcal{O}^{2}}$. Note that one is largely free in the ordering of the different parts of this operator; merely the relative ordering of the $\beta$ and $\mathcal{O}$ factors in the numerator matters. Otherwise, the different parts commute. Further more, $\epsilon$ is positive definite, so there are no problems defining square roots and inverses. One can easily check unitarity, $U^{\dagger} U=1$, and also

$$
U^{\dagger}(\beta m+\mathcal{O}) U=\beta \epsilon=\beta m+\beta(\epsilon-m) .
$$

Hence, one has succeeded in eliminating the odd term $\mathcal{O}$ in the Hamiltonian in favor of the even term $\beta(\epsilon-m)$; the new Hamiltonian now reads

$$
H^{\prime}=\beta m+\beta(\epsilon-m)+U^{\dagger}\left(-i \frac{\partial U}{\partial t}\right)+U^{\dagger} \mathcal{E} U
$$

However, the other two terms in $H^{\prime}$ may reintroduce new odd terms, i.e., in general, this transformation is not exact. Nonetheless, if $\mathcal{O}$ and $\mathcal{E}$ are in some sense small, i.e., if one is content with a power expansion, the induced new odd terms may be of higher order, and it will be sufficient to iterate the transformation a finite number of times, until the remaining odd terms are of sufficiently high order to be dropped. For present purposes, it will be necessary to keep only terms of up to second order in $\mathcal{E}$, or second order in $\partial / \partial t$, or 
altogether fourth order in the objects $\mathcal{E}, \mathcal{O}, \partial / \partial t$. This specification will be justified below as more concrete expressions become available from which one can read off the required order. Also the second derivative $\ddot{\mathcal{O}}$ can be dropped. Note that, here and in the following, the dot denotes the partial derivative $\partial / \partial t$. Expanding

$$
\begin{gathered}
\epsilon=m\left(1+\frac{1}{2} \frac{\mathcal{O}^{2}}{m^{2}}-\frac{1}{8} \frac{\mathcal{O}^{4}}{m^{4}}+\cdots\right) \\
U=1-\frac{1}{2 m} \beta \mathcal{O}-\frac{1}{8 m^{2}} \mathcal{O}^{2}+\frac{3}{16 m^{3}} \beta \mathcal{O}^{3}+\frac{11}{128 m^{4}} \mathcal{O}^{4}+\cdots \\
U^{\dagger}\left(-i \frac{\partial U}{\partial t}\right)=\frac{i}{2 m} \beta \dot{\mathcal{O}}+\frac{i}{8 m^{2}}[\dot{\mathcal{O}}, \mathcal{O}] \\
-\frac{i}{16 m^{3}} \beta\left(3 \mathcal{O}^{2} \dot{\mathcal{O}}+2 \mathcal{O} \dot{\mathcal{O}} \mathcal{O}+3 \dot{\mathcal{O}} \mathcal{O}^{2}\right)+\cdots \\
U^{\dagger} \mathcal{E} U=\mathcal{E}+\frac{1}{2 m} \beta[\mathcal{O}, \mathcal{E}]+\frac{1}{8 m^{2}}[[\mathcal{O}, \mathcal{E}], \mathcal{O}] \\
+\frac{1}{16 m^{3}} \beta\left(3\left[\mathcal{E}, \mathcal{O}^{3}\right]+[\mathcal{O}, \mathcal{O} \mathcal{E} \mathcal{O}]\right)+\cdots
\end{gathered}
$$

and classifying the terms with respect to their even/odd character, the new Hamiltonian is

$$
H^{\prime}=\beta m+\mathcal{E}^{\prime}+\mathcal{O}^{\prime}
$$

with

$$
\begin{aligned}
\mathcal{E}^{\prime}= & \frac{1}{2 m} \beta \mathcal{O}^{2}-\frac{1}{8 m^{3}} \beta \mathcal{O}^{4}+\frac{i}{8 m^{2}}[\dot{\mathcal{O}}, \mathcal{O}]+\mathcal{E} \\
& +\frac{1}{8 m^{2}}[[\mathcal{O}, \mathcal{E}], \mathcal{O}] \\
\mathcal{O}^{\prime}= & \frac{i}{2 m} \beta \dot{\mathcal{O}}-\frac{i}{16 m^{3}} \beta\left(3 \mathcal{O}^{2} \dot{\mathcal{O}}+2 \mathcal{O} \dot{\mathcal{O}} \mathcal{O}+3 \dot{\mathcal{O}} \mathcal{O}^{2}\right) \\
& +\frac{1}{2 m} \beta[\mathcal{O}, \mathcal{E}]+\frac{1}{16 m^{3}} \beta\left(3\left[\mathcal{E}, \mathcal{O}^{3}\right]+[\mathcal{O}, \mathcal{O} \mathcal{E} \mathcal{O}]\right) .
\end{aligned}
$$

The odd term $\mathcal{O}^{\prime}$ now starts at one order higher in $\mathcal{E}, \mathcal{O}, \partial / \partial t$ than the original $\mathcal{O}$. This is not yet sufficient to preclude additional contributions to the even term up to the desired order upon further iteration. Repeating the procedure, one has the new Hamiltonian

$$
H^{\prime \prime}=\beta m+\mathcal{E}^{\prime \prime}+\mathcal{O}^{\prime \prime}
$$

with

$$
\begin{aligned}
\mathcal{E}^{\prime \prime}= & \frac{1}{2 m} \beta \mathcal{O}^{\prime 2}-\frac{1}{8 m^{3}} \beta \mathcal{O}^{\prime 4}+\frac{i}{8 m^{2}}\left[\dot{\mathcal{O}}^{\prime}, \mathcal{O}^{\prime}\right]+\mathcal{E}^{\prime} \\
& +\frac{1}{8 m^{2}}\left[\left[\mathcal{O}^{\prime}, \mathcal{E}^{\prime}\right], \mathcal{O}^{\prime}\right] \\
= & \frac{1}{2 m} \beta \mathcal{O}^{2}-\frac{1}{8 m^{3}} \beta \mathcal{O}^{4}+\frac{i}{8 m^{2}}[\dot{\mathcal{O}}, \mathcal{O}]+\mathcal{E}+\frac{1}{8 m^{2}}[[\mathcal{O}, \mathcal{E}], \mathcal{O}] \\
& +\frac{1}{8 m^{3}} \beta \dot{\mathcal{O}}^{2}-\frac{1}{8 m^{3}} \beta[\mathcal{O}, \mathcal{E}]^{2}-\frac{i}{8 m^{3}} \beta \dot{\mathcal{O}}[\mathcal{O}, \mathcal{E}] \\
& -\frac{i}{8 m^{3}} \beta[\mathcal{O}, \mathcal{E}] \dot{\mathcal{O}} \\
\mathcal{O}^{\prime \prime}= & \frac{i}{2 m} \beta \dot{\mathcal{O}}^{\prime}-\frac{i}{16 m^{3}} \beta\left(3 \mathcal{O}^{\prime 2} \dot{\mathcal{O}}^{\prime}+2 \mathcal{O}^{\prime} \dot{\mathcal{O}}^{\prime} \mathcal{O}^{\prime}+3 \dot{\mathcal{O}}^{\prime} \mathcal{O}^{\prime 2}\right) \\
& +\frac{1}{2 m} \beta\left[\mathcal{O}^{\prime}, \mathcal{E}^{\prime}\right]+\frac{1}{16 m^{3}} \beta\left(3\left[\mathcal{E}^{\prime}, \mathcal{O}^{\prime 3}\right]+\left[\mathcal{O}^{\prime}, \mathcal{O}^{\prime} \mathcal{E}^{\prime} \mathcal{O}^{\prime}\right]\right) \\
= & \frac{i}{2 m^{2}}[\dot{\mathcal{O}}, \mathcal{E}]+\frac{i}{4 m^{2}}[\mathcal{O}, \dot{\mathcal{E}}]-\frac{i}{8 m^{3}} \beta\left(\mathcal{O}^{2} \dot{\mathcal{O}}+\dot{\mathcal{O}}^{2}\right) \\
& -\frac{1}{4 m^{2}}[\mathcal{E},[\mathcal{O}, \mathcal{E}]]-\frac{1}{8 m^{3}} \beta\left(\mathcal{O}^{2}[\mathcal{O}, \mathcal{E}]+[\mathcal{O}, \mathcal{E}] \mathcal{O}^{2}\right)
\end{aligned}
$$

The odd term $\mathcal{O}^{\prime \prime}$ now starts at third order in $\mathcal{E}, \mathcal{O}, \partial / \partial t$. This means that subsequent iterations will only contribute new terms to the even part that are of too high order to be retained, while successively increasing the order of the odd part until it can be completely dropped. Thus, to the desired order, the final Foldy-Wouthuysen Hamiltonian has been obtained:

$$
\begin{aligned}
H_{\mathrm{FW}}= & \beta m+\mathcal{E}^{\prime \prime} \\
= & \beta m+\frac{1}{2 m} \beta \mathcal{O}^{2}-\frac{1}{8 m^{3}} \beta \mathcal{O}^{4}+\frac{i}{8 m^{2}}[\dot{\mathcal{O}}, \mathcal{O}]+\mathcal{E} \\
& +\frac{1}{8 m^{2}}[[\mathcal{O}, \mathcal{E}], \mathcal{O}]+\frac{1}{8 m^{3}} \beta \dot{\mathcal{O}}^{2}-\frac{1}{8 m^{3}} \beta[\mathcal{O}, \mathcal{E}]^{2} \\
& -\frac{i}{8 m^{3}} \beta(\dot{\mathcal{O}}[\mathcal{O}, \mathcal{E}]+[\mathcal{O}, \mathcal{E}] \dot{\mathcal{O}}) .
\end{aligned}
$$

\section{B. Evaluation in terms of background fields and momenta}

The Dirac Hamiltonian for the neutron with anomalous magnetic moment $\mu$ is of the form (6), with, cf. (4),

$$
\mathcal{O}=\alpha \cdot p+i \mu \gamma \cdot E, \quad \mathcal{E}=-\mu \Pi \cdot B
$$

Here, the position representation is adopted, where $p=-i \nabla$, and the Dirac representation is used, in which

$$
\begin{aligned}
\beta & =\left(\begin{array}{cc}
1 & 0 \\
0 & -1
\end{array}\right), \quad \alpha^{i}=\left(\begin{array}{cc}
0 & \sigma^{i} \\
\sigma^{i} & 0
\end{array}\right), \quad \gamma^{i}=\beta \alpha^{i}, \\
\Pi^{i} & =\beta \sigma^{i}, \quad \gamma^{5}=\left(\begin{array}{ll}
0 & 1 \\
1 & 0
\end{array}\right)
\end{aligned}
$$


with $\sigma^{i}$ denoting the Pauli matrices. The goal is to present the Foldy-Wouthuysen Hamiltonian $H_{\mathrm{FW}}$ in a form in which:

(i) All momentum operators $p$ have been commuted through to the right.

(ii) The Dirac structures have been simplified to a manifestly block-diagonal form, upon which $H_{\mathrm{FW}}$ can be restricted to the upper components, leaving at most Pauli matrix structures.

(iii) Terms higher than quadratic in the external fields $E$, $B$ have been dropped.

(iv) Terms containing higher than first derivatives of external fields have been dropped.

(v) Terms of higher than altogether fourth order in the objects $E, B, \partial / \partial t, \nabla, p$ have been dropped (where $\partial / \partial t, \nabla$ are always acting only on a specific external field, whereas $p$ stands as an operator on its own).

(vi) At most one power of $p$ is kept in terms already quadratic in the external fields $E, B$.

(vii) At most three powers of $p$ are kept in terms linear in the external fields $E, B$.

Again, the reasoning leading to this specification will become fully apparent below as the treatment unfolds; of course, the third and fourth points are already clear from the stated objectives of the calculation, i.e., expansion to second order in the external fields, up to first derivatives of those fields. Note that this specification justifies the truncation imposed in the previous section, as stated after Eq. (13): Since $\mathcal{E}$ is proportional to $B$, only up to second order in $\mathcal{E}$ is required; since only one derivative each of at most two external fields is allowed, only up to second order in $\partial / \partial t$ is required; and since both $\mathcal{E}$ and $\mathcal{O}$ each supply one order in the objects $E, B, \nabla, p$, only up to fourth order in $\mathcal{E}$, $\mathcal{O}, \partial / \partial t$ is required.

Treating the terms appearing in (24) in turn, one has

$$
\begin{aligned}
\mathcal{O}^{2}= & -\mu \nabla \cdot E-i \mu \sigma \cdot(\nabla \times E)+\mu^{2} E^{2} \\
& -2 \mu \sigma \cdot(E \times p)+p^{2},
\end{aligned}
$$

where, having restricted to the upper components, one can set $\beta=1$. Also, here and in the following, the derivative $\nabla$ only acts on the field immediately to its right, whereas the momentum operator $p=-i \nabla$ acts on all objects to its right. The term $\mathcal{O}^{4}$, which is the most complex one appearing in $H_{\mathrm{FW}}$, can be obtained by squaring (27),

$$
\begin{aligned}
\mathcal{O}^{4}= & -\mu^{2} \frac{1}{2}\left(\nabla_{i} E_{j}+\nabla_{j} E_{i}\right)^{2}-\mu^{2}\left(\nabla_{i} E_{j}-\nabla_{j} E_{i}\right)^{2}+\mu^{2}(\nabla \cdot E)^{2}+2 \mu^{2} i \sigma \cdot(\nabla \times E)(\nabla \cdot E)+4 \mu^{2} \sigma_{j} E_{i} \nabla_{j} E_{l} \epsilon_{i l m} p_{m} \\
& -4 \mu^{2}(\sigma \cdot E)(\nabla \times E) \cdot p-12 \mu^{2} i E_{i} \nabla_{j} E_{i} p_{j}+4 \mu^{2} i E_{i} \nabla_{i} E_{j} p_{j}+4 \mu^{2}(\nabla \cdot E) \sigma \cdot(E \times p)+4 \mu^{2} i(\nabla \cdot E) E \cdot p \\
& +4 \mu i \sigma_{k} \epsilon_{k i j} \nabla_{l} E_{i} p_{l} p_{j}-2 \mu i \sigma \cdot(\nabla \times E) p^{2}-2 \mu(\nabla \cdot E) p^{2}-4 \mu \sigma \cdot(E \times p) p^{2}+p^{4} .
\end{aligned}
$$

Arriving at this result requires only standard, if lengthy, Pauli matrix and $\epsilon$-symbol algebra, apart, perhaps, from the not immediately apparent identity $(\nabla \times E)_{j}\left(\nabla_{i} E_{j}-\nabla_{j} E_{i}\right)=0$. One furthermore has

$$
\begin{gathered}
{[\dot{\mathcal{O}}, \mathcal{O}]=-2 \mu^{2} i \sigma \cdot(E \times \dot{E})+2 \mu i \dot{E} \cdot p} \\
\dot{\mathcal{O}}^{2}=\mu^{2} \dot{E}^{2} .
\end{gathered}
$$

Turning to the terms containing $\mathcal{E}$, apart from the original even operator

$$
\mathcal{E}=-\mu \sigma \cdot B
$$

all other terms involve the intermediate odd operator

$$
[\mathcal{O}, \mathcal{E}]=\mu \beta \gamma^{5} \sigma \cdot(\nabla \times B)+2 \mu \beta \gamma^{5} B \cdot p+2 \mu^{2} i \gamma^{5} E \cdot B,
$$

where Maxwell's equations for the external fields have been used to drop $\nabla \cdot B=0$. From this, one obtains

$$
\begin{gathered}
{[[\mathcal{O}, \mathcal{E}], \mathcal{O}]=-\mu^{2} E_{j} \sigma_{i}\left(3\left(\nabla_{i} B_{j}-\nabla_{j} B_{i}\right)+\left(\nabla_{i} B_{j}+\nabla_{j} B_{i}\right)\right)} \\
-2 \mu^{2} B_{i} \sigma_{j}\left(\nabla_{i} E_{j}+\nabla_{j} E_{i}\right)+2 \mu(\nabla \times B) \cdot p \\
-2 \mu i \sigma_{j} \nabla_{j} B_{i} p_{i}+4 \mu \sigma_{j} B_{i} p_{j} p_{i} \\
{[\mathcal{O}, \mathcal{E}]^{2}=-\mu^{2}(\nabla \times B)^{2}+4 \mu^{2} i B_{i} \nabla_{i} B_{j} p_{j}} \\
-4 \mu^{2} \sigma \cdot(\nabla \times B) B \cdot p \\
\dot{\mathcal{O}}[\mathcal{O}, \mathcal{E}]+[\mathcal{O}, \mathcal{E}] \dot{\mathcal{O}}=-2 \mu^{2} i(\nabla \times B) \cdot \dot{E}-4 \mu^{2} i(\sigma \cdot \dot{E}) B \cdot p .
\end{gathered}
$$


Inserting (27)-(35) into (24) yields the Foldy-Wouthuysen Hamiltonian expressed in terms of background fields and momenta. For further use, it is convenient to gather the aggregate expression, ${ }^{2}$

$$
\begin{aligned}
H_{\mathrm{FW}}= & m-\mu \sigma \cdot B+\frac{1}{2 m}\left(-\mu \nabla \cdot E-i \mu \sigma \cdot(\nabla \times E)+\mu^{2} E^{2}-2 \mu \sigma \cdot(E \times p)+p^{2}\right)+\frac{1}{8 m^{3}} \mu^{2} \dot{E}^{2} \\
& -\frac{1}{8 m^{3}}\left(-\mu^{2} \frac{1}{2}\left(\nabla_{i} E_{j}+\nabla_{j} E_{i}\right)^{2}-\mu^{2}\left(\nabla_{i} E_{j}-\nabla_{j} E_{i}\right)^{2}+\mu^{2}(\nabla \cdot E)^{2}+2 \mu^{2} i \sigma \cdot(\nabla \times E)(\nabla \cdot E)\right. \\
& +4 \mu^{2} \sigma_{j} E_{i} \nabla_{j} E_{l} \epsilon_{i l m} p_{m}-4 \mu^{2}(\sigma \cdot E)(\nabla \times E) \cdot p-12 \mu^{2} i E_{i} \nabla_{j} E_{i} p_{j}+4 \mu^{2} i E_{i} \nabla_{i} E_{j} p_{j} \\
& +4 \mu^{2}(\nabla \cdot E) \sigma \cdot(E \times p)+4 \mu^{2} i(\nabla \cdot E) E \cdot p+4 \mu i \sigma_{k} \epsilon_{k i j} \nabla_{l} E_{i} p_{l} p_{j}-2 \mu i \sigma \cdot(\nabla \times E) p^{2} \\
& \left.-2 \mu(\nabla \cdot E) p^{2}-4 \mu \sigma \cdot(E \times p) p^{2}+p^{4}\right) \\
& +\frac{1}{8 m^{2}}\left(2 \mu^{2} \sigma \cdot(E \times \dot{E})-2 \mu \dot{E} \cdot p\right)-\frac{1}{8 m^{3}}\left(2 \mu^{2}(\nabla \times B) \cdot \dot{E}+4 \mu^{2}(\sigma \cdot \dot{E}) B \cdot p\right) \\
& +\frac{1}{8 m^{2}}\left(-\mu^{2} E_{j} \sigma_{i}\left(3\left(\nabla_{i} B_{j}-\nabla_{j} B_{i}\right)+\left(\nabla_{i} B_{j}+\nabla_{j} B_{i}\right)\right)-2 \mu^{2} B_{i} \sigma_{j}\left(\nabla_{i} E_{j}+\nabla_{j} E_{i}\right)\right. \\
& \left.+2 \mu(\nabla \times B) \cdot p-2 \mu i \sigma_{j} \nabla_{j} B_{i} p_{i}+4 \mu \sigma_{j} B_{i} p_{j} p_{i}\right) \\
& -\frac{1}{8 m^{3}}\left(-\mu^{2}(\nabla \times B)^{2}+4 \mu^{2} i B_{i} \nabla_{i} B_{j} p_{j}-4 \mu^{2} \sigma \cdot(\nabla \times B) B \cdot p\right) .
\end{aligned}
$$

\section{ENERGY OF ZERO-MOMENTUM NEUTRON STATES}

\section{A. Zero momentum and local limit}

In the Foldy-Wouthuysen Hamiltonian (36), the electric and magnetic fields are still functions of space and time. As already indicated in the preliminary discussion at the end of Sec. I, one must take care in reducing this to a result of the form (1) for the energy of a zero-momentum neutron, in which the fields and their derivatives are taken to be local constants at the position of a localized neutron state. For any finite neutron mass, the neutron wave function must feature a residual extent to allow the neutron momentum to be bounded to negligible magnitudes. This residual extent probes the external field in the neighborhood of the neutron, leading to corrections to the neutron energy compared to the one obtained by simply replacing all fields in (36) directly by their local values. It should be emphasized that these corrections depend on further details of the environment in which one places the neutron, which influence its spatial wave function. They are therefore not purely intrinsic properties of the neutron that could be discussed in full generality; instead, they require a model for the environment.

\footnotetext{
${ }^{2}$ The difference in sign between the term $-\frac{1}{2 m} \mu \nabla \cdot E$ in (36) and the corresponding term in [55] should be noted. This difference can be traced back to a corresponding difference in sign of the electric field term in the original Dirac Hamiltonian (4) compared to the one used in [55]. To compare expressions between here and [55], one must change the sign of the magnetic moment $\mu$; Foldy contributions to polarizabilities, which are proportional to $\mu^{2}$, are not affected.
}

Thus, in order to exemplify these effects, in the detailed evaluation in Sec. III B, the neutron will be placed in a box with periodic boundary conditions, with the goal of ultimately expressing the neutron energy in terms of the values of the external fields at the center of the box. This choice of model is relevant, e.g., for the analysis of lattice QCD calculations of neutron energies; furthermore, it allows one to maintain the notion of an exact zero-momentum state, i.e., one can simply set $p=0$ when applying (36) to such a state. The $p=0$ state moreover is symmetric about the center of the box, such that, placing the origin at the center of the box, one has $\left\langle p=0\left|x_{i}\right| p=0\right\rangle=0$ in any direction $i$; this reduces the number of corrections that must be considered. In fact, only two terms in (36) require closer scrutiny to the order being pursued here, namely, the terms $-\mu \sigma \cdot B$ and $\frac{1}{2 m} \mu^{2} E^{2}$. Expanding the spatial dependence of the fields to linear order around the origin, one has

$$
\begin{array}{r}
-\mu \sigma \cdot B(x, t)=-\mu \sigma \cdot B-\mu \sigma_{i}\left(\nabla_{j} B_{i}\right) x_{j}+\cdots \\
\frac{\mu^{2}}{2 m}(E(x, t))^{2}=\frac{\mu^{2}}{2 m}\left(E^{2}+\left(\nabla_{j} E_{i} \nabla_{k} E_{i}\right) x_{j} x_{k}\right)+\cdots
\end{array}
$$

where fields without space-time arguments are evaluated at the origin (the temporal dependence will be commented upon presently). The space-dependent terms on the righthand sides of (37) and (38) spoil the eigenstate character of the $p=0$ state. Their effects can, however, be taken into account in perturbation theory around the $p=0$ state. This will be pursued in Sec. III B. 
In addition to their spatial dependence, the external fields also are time dependent. The time dependence must be treated on a different footing than the spatial dependence: As far as the latter is concerned, there is a direct tension between the localization assumption and using a $p=0$ state, with the uncertainty relation limiting the accuracy to which both of these specifications can be maintained. Consequently, taking the spatial dependences in (37) and (38) into account perturbatively cannot be avoided. On the other hand, there is no analogous direct clash in the temporal direction; if the temporal dependence of the external fields is sufficiently slow, an adiabatic treatment becomes applicable, in which transitions to excited states are absent and the fields can indeed simply be evaluated at the expansion point $t=0$. In fact, on the contrary, such an adiabatic treatment is the furthest one can go while remaining consistent with the notion of a state with a well-defined energy embodied in the initial form (1) under consideration. Once transitions to excited states are induced via time-dependent perturbation theory, the form (1) loses its meaning.

In summary, the temporal evolution will be assumed to be adiabatic; the evaluation of the spatial perturbative corrections is deferred to the next section. Before taking these corrections into account, one obtains the neutron energy in a zero-momentum state by simply setting $p=0$ in the Foldy-Wouthuysen Hamiltonian (36),

$$
\begin{aligned}
W_{\mathrm{FW}}^{p=0}= & m-\mu \sigma \cdot B-\frac{\mu}{2 m} i \sigma \cdot(\nabla \times E)-\frac{\mu}{2 m} \nabla \cdot E+\frac{\mu^{2}}{2 m} E^{2}+\left[\frac{\mu^{2}}{4 m^{2}} \sigma \cdot(E \times \dot{E})-\frac{3 \mu^{2}}{8 m^{2}} \sigma \cdot(E \times(\nabla \times B))\right] \\
& -\frac{\mu^{2}}{8 m^{2}} \sigma_{i} E_{j}\left(\nabla_{i} B_{j}+\nabla_{j} B_{i}\right)-\frac{\mu^{2}}{4 m^{2}} \sigma_{i} B_{j}\left(\nabla_{i} E_{j}+\nabla_{j} E_{i}\right)+\frac{\mu^{2}}{16 m^{3}}\left(\nabla_{i} E_{j}+\nabla_{j} E_{i}\right)^{2}+\frac{\mu^{2}}{4 m^{3}}(\nabla \times E)^{2} \\
& +\left[\frac{\mu^{2}}{8 m^{3}} \dot{E}^{2}+\frac{\mu^{2}}{8 m^{3}}(\nabla \times B)^{2}-\frac{\mu^{2}}{4 m^{3}}(\nabla \times B) \cdot \dot{E}\right]-\frac{\mu^{2}}{8 m^{3}}(\nabla \cdot E)^{2}-\frac{\mu^{2}}{4 m^{3}} i \sigma \cdot(\nabla \times E)(\nabla \cdot E) .
\end{aligned}
$$

It is clear that the specification of terms to be kept given at the beginning of Sec. II B is sufficient to guarantee that (39) is complete to the desired order, i.e., up to second order in the external fields, with up to first derivatives of those fields: All terms up to second order in the fields were kept; in the $p=0$ case, only terms containing no $p$ operators are relevant; from among these, only terms up to altogether fourth order in $E, B, \partial / \partial t, \nabla$ are needed, since each of the at most two external fields can absorb at most one derivative.

In the form (39), it has not yet been assumed that electric charges or currents are absent; it includes, e.g., the $\nabla \cdot E$ structure that embodies the influence of an external electric charge density which enters effects such as the Darwin term. If one specializes to the vacuum, the terms grouped in square brackets can be combined using the vacuum Maxwell equations for the external fields,

$\nabla \cdot E=0, \quad \nabla \cdot B=0, \quad \nabla \times E=-\dot{B}, \quad \nabla \times B=\dot{E}$

yielding

$$
\begin{aligned}
W_{\mathrm{FW}}^{p=0}= & m-\mu \sigma \cdot B+\frac{\mu}{2 m} i \sigma \cdot \dot{B}+\frac{\mu^{2}}{2 m} E^{2} \\
& -\frac{\mu^{2}}{8 m^{2}} \sigma \cdot(E \times \dot{E})-\frac{\mu^{2}}{8 m^{2}} \sigma_{i} E_{j}\left(\nabla_{i} B_{j}+\nabla_{j} B_{i}\right) \\
& -\frac{\mu^{2}}{4 m^{2}} \sigma_{i} B_{j}\left(\nabla_{i} E_{j}+\nabla_{j} E_{i}\right) \\
& +\frac{\mu^{2}}{16 m^{3}}\left(\nabla_{i} E_{j}+\nabla_{j} E_{i}\right)^{2}+\frac{\mu^{2}}{4 m^{3}} \dot{B}^{2} .
\end{aligned}
$$

The terms quadratic in the external fields can now be directly compared to the form of the effective Hamiltonian defining the corresponding polarizabilities. They constitute the Foldy contributions that have to be separated out in the effective Hamiltonian to isolate the proper polarizabilities that arise as a consequence of the extended, composite character of the neutron. These results generalize the wellknown Foldy term that is associated specifically with the $E^{2}$ structure.

\section{B. Perturbative corrections to the local limit}

It remains to evaluate the perturbative corrections to (39) or (41) due to the spatially dependent terms on the right-hand sides of (37) and (38). As motivated in the preceding section, this will be accomplished in a model setting where the neutron is placed in a box $[-L / 2, L / 2]^{3}$ with periodic boundary conditions. The unperturbed Hamiltonian is taken to be the term $H^{0}=p^{2} /(2 m)$ from (36). The unperturbed spatial wave functions are

$$
\psi_{\vec{n}}^{0}(\vec{x})=\frac{1}{L^{3 / 2}} e^{i 2 \pi \vec{n} \cdot \vec{x} / L}
$$

with integer $n_{i}$ and energies $W_{\vec{n}}^{0}=2 \pi^{2}(\vec{n})^{2} /\left(m L^{2}\right)$. For the following, it is not necessary to specify a spin quantization axis, since the perturbations are proportional to the unit matrix in the $p=0$ subspace; one could, e.g., choose to diagonalize (39) to completely decouple the entire Hamiltonian in that subspace. Starting with the electric field term from (38), 


$$
H^{E}=\frac{\mu^{2}}{2 m}\left(\nabla_{j} E_{i} \nabla_{k} E_{i}\right) x_{j} x_{k}
$$

first-order perturbation theory is sufficient, yielding

$$
W^{E, 1}=\left\langle p=0\left|H^{E}\right| p=0\right\rangle=(m L)^{2} \frac{\mu^{2}}{24 m^{3}} \nabla_{j} E_{i} \nabla_{j} E_{i} .
$$

Note that the strength of this contribution relative to analogous terms in (39) or (41) is controlled by the parameter $m L$, making explicit the dependence of these perturbative corrections on model assumptions about the environment. Additional remarks on this point follow further below. The magnetic field term from (37),

$$
H^{B}=-\mu \sigma_{i}\left(\nabla_{j} B_{i}\right) x_{j},
$$

on the other hand requires second-order perturbation theory (it does not contribute at first order; indeed, $\left.H^{B}\right|_{p=0}=0$ ). Summed over a complete set of intermediate spins $S$, the moduli squared of the transition matrix elements are

$$
\sum_{S}\left|\left\langle\vec{n}, S\left|H^{B}\right| p=0\right\rangle\right|^{2}=\sum_{j} \mu^{2}\left(\nabla_{j} B_{i} \nabla_{j} B_{i}\right)\left(\frac{L}{2 \pi n_{j}}\right)^{2} \delta_{\vec{n}, n_{j} \vec{e}_{j}}
$$

and hence, the resulting perturbative correction is

$$
\begin{aligned}
W^{B, 2} & =\sum_{\vec{n} \neq 0, S} \frac{\left|\left\langle\vec{n}, S\left|H^{B}\right| p=0\right\rangle\right|^{2}}{W_{0}^{0}-W_{\vec{n}}^{0}} \\
& =-(m L)^{4} \frac{\mu^{2}}{360 m^{3}} \nabla_{j} B_{i} \nabla_{j} B_{i}
\end{aligned}
$$

with a strength again controlled by the parameter $m L$.

Of course, the formal limit $m L \rightarrow 0$ in which the correction terms (44) and (47) vanish is not compatible with the notion of a well-defined one-particle neutron state. A natural lower bound is provided by equating $L$ with the Compton wavelength, i.e., $m L=2 \pi$. On the other hand, the derivative expansion employed here is best applicable when keeping $L$ as small as possible. In typical lattice QCD calculations, $m L$ ranges between values of about 10-30; in this range, the perturbative effects (44) and (47) are considerably stronger than the analogous terms in (39) or (41). This also remains the case if one replaces the nonrelativistic unperturbed energies in (47) with their relativistic counterparts; at $m L=2 \pi$, the result (47) is then enhanced by a factor 1.25 , and the factor approaches unity with rising $m L$. Of course, in any complete analysis of, e.g., a lattice QCD calculation, these perturbative corrections constitute only one among many systematic effects that must be accounted for in order to extract physical results.

\section{NEUTRON AT ZERO VELOCITY}

\section{A. Velocity operator}

Since, in a gauge theory, zero momentum is not synonymous with zero velocity, it is interesting to consider separately the energy of a neutron at rest in the sense of being in a zero-velocity state $[55,60]$. The velocity operator is defined as the total time derivative of the position operator, which can be obtained from the commutator of the position with the Hamiltonian,

$$
v_{n}=-i\left[x_{n}, H_{\mathrm{FW}}\right]=\frac{\partial H_{\mathrm{FW}}}{\partial p_{n}} .
$$

Note that the position operator commutes with the external fields, which are treated as given functions of position and time.

The concept of a zero-velocity state is not unproblematic. The starting point of the following discussion is the assumption that there is a state $|v=0\rangle$ that is annihilated by all components of the velocity operator, $v_{n}|v=0\rangle=0$, and explore how far this assumption carries. As one might already suspect from (48), and as will become more clear from the explicit expressions below, the individual components $v_{n}$ do not in general commute, and therefore one cannot in general expect to construct simultaneous eigenstates of these operators. However, this by itself does not exclude the possibility of a state with, specifically, $v_{n}|v=0\rangle=0$, similar to the case of the standard angular momentum algebra, for which one does have $J_{n}|j=0\rangle=0$. No attempt will be made to construct a state $|v=0\rangle$ explicitly. Rather, to the extent that such a state exists, the results obtained will apply to it.

Certainly, although the concept of a zero-velocity neutron is a priori physically meaningful, such a state can in general only be of a transitory nature in the presence of external electromagnetic fields. Already at the classical level, a magnetic moment is accelerated in an inhomogeneous magnetic field. In that case, one can only expect a consistent treatment in terms of such a state up to a limited order in a derivative expansion such as pursued in this work. To the order considered here, however, no inconsistency related to the inhomogeneity of the magnetic field will become apparent.

On the other hand, a zero-velocity quantum state presumably requires a certain spatial extension, and, as already discussed in previous sections, a derivative expansion of the external fields can only capture a limited range of spatial behavior. This likewise may manifest itself in inconsistencies beyond a certain order in the derivative expansion. Such an inconsistency will in fact emerge in the treatment below, specifically with respect to the terms in the neutron mass proportional to $\nabla_{i} E_{j} \nabla_{i} E_{j}$ and $(\nabla \cdot E)^{2}$. The coefficients of these terms will be seen to be ambiguous, and no completely cogent scheme has become apparent within the calculational framework employed here that would permit a definite 
determination of these terms. Presumably, this would require going beyond the derivative expansion (1) from the very beginning.

An alternative definition of a zero-velocity state can be contemplated in which one considers only the velocity squared, and one assumes only the existence of a state $\left|v^{2}=0\right\rangle$ with $v^{2}\left|v^{2}=0\right\rangle=0$. This will likewise be explored further below; while it does circumvent the issue of the individual components $v_{n}$ not commuting, this approach has its own difficulties and does not finally resolve the aforementioned ambiguity.

In [60], a definition of a zero-velocity state is considered for which only the considerably weaker condition $\langle v\rangle=0$ is posited. Such a treatment requires a construction of the state, which is beyond the scope of the present work. It should be noted, however, that also this definition does not eliminate the transitory nature of a zero-velocity state. Taken by itself, this condition would encompass generic bound states, presumably including highly excited ones that may be interpreted in terms of classical orbits. In that limit, it becomes questionable whether the neutron can still be viewed as being truly at rest, and it may be necessary to supply a supplementary characterization of the states to be considered.

For $H_{\mathrm{FW}}$ given by (36), the velocity operator takes the form

$$
v_{n}=\frac{1}{m} p_{n}-\frac{1}{2 m^{3}} p^{2} p_{n}+G_{n},
$$

where $G_{n}$ summarizes all the terms that are at least linear in the external fields,

$$
\begin{aligned}
G_{n}= & -\frac{\mu}{m}(\sigma \times E)_{n}+\frac{\mu}{4 m^{2}}\left(-\dot{E}_{n}+(\nabla \times B)_{n}-i \sigma_{i} \nabla_{i} B_{n}\right)+\frac{\mu^{2}}{2 m^{3}}\left(-\sigma_{j} E_{i} \nabla_{j} E_{k} \epsilon_{i k n}+(\sigma \cdot E)(\nabla \times E)_{n}+3 i E_{i} \nabla_{n} E_{i}\right. \\
& \left.-i E_{i} \nabla_{i} E_{n}-(\nabla \cdot E)(\sigma \times E)_{n}-i(\nabla \cdot E) E_{n}-i B_{i} \nabla_{i} B_{n}+\sigma \cdot(\nabla \times B) B_{n}-(\sigma \cdot \dot{E}) B_{n}\right) \\
& +\frac{\mu}{2 m^{3}}\left(-i \sigma_{j} \epsilon_{j i n} \nabla_{k} E_{i} p_{k}-i \sigma_{k} \epsilon_{k i j} \nabla_{n} E_{i} p_{j}+i \sigma \cdot(\nabla \times E) p_{n}+(\nabla \cdot E) p_{n}\right) \\
& +\frac{\mu}{2 m^{2}}\left(\sigma_{i} B_{n} p_{i}+\sigma_{n} B \cdot p\right)+\frac{\mu}{2 m^{3}}\left(2 \sigma \cdot(E \times p) p_{n}+(\sigma \times E)_{n} p^{2}\right) .
\end{aligned}
$$

\section{B. Action of Foldy-Wouthuysen Hamiltonian on a $|v=0\rangle$ state}

To derive the action of $H_{F W}$ on a zero-velocity state $|v=0\rangle$, the momentum operator can be eliminated in favor of the velocity operator using the following iterative scheme. Rearranging (49) into

$$
\begin{aligned}
p_{n} & =\left(1-\frac{p^{2}}{2 m^{2}}\right)^{-1} m\left(v_{n}-G_{n}\right) \\
& =\left(1+\frac{p^{2}}{2 m^{2}}+\cdots\right) m\left(v_{n}-G_{n}\right),
\end{aligned}
$$

where higher orders in $p$ can be neglected in the right-hand expression, it follows that the action of $p_{n}$ on a zerovelocity state $|v=0\rangle$ yields

$$
p_{n}=-m\left(1+\frac{p^{2}}{2 m^{2}}\right) G_{n}
$$

thus generating at least one power of an external field. With $H_{F W}$ given by (36), i.e., with all momentum operators commuted through to the right, one can let the rightmost momentum operator act on the zero-velocity state and therefore substitute it with (52). This yields an expression in which the momentum operators are not all ordered to the right; one then has to commute all momentum operators to the right again. At that point, one can iterate the procedure, i.e., again let the rightmost momentum operator act on the zero-velocity state, etc. This procedure has to be performed at most twice, since every iteration generates an additional power of an external field.

Before proceeding, it is convenient at this point to revisit the specification given at the beginning of Sec. II B of the terms that were to be kept in deriving the Foldy-Wouthuysen Hamiltonian (36). Consider first terms quadratic in the external fields. To begin with, as far as $H_{\mathrm{FW}}$ itself is concerned, no additional factor of $p$ (which, as always, would be ordered to the right) needs to be taken into account, since such a factor of $p$, applied to the zero-velocity state, would generate an additional external field. However, one must also ensure that terms in $G_{n}$ are kept to sufficiently high order; deriving $G_{n}$ from $H_{\mathrm{FW}}$ removes one factor of $p$. As a result, also terms in $H_{\mathrm{FW}}$ quadratic in the external fields and linear in $p$ must be kept, since the corresponding term in $G_{n}$ is then simply quadratic in the external fields with no further factors of $p$. However, if there were yet one more factor of $p$, then $G_{n}$ would be quadratic in the external fields with an additional factor $p$ which, acting on the zero-velocity state, would generate an additional external field. Such terms can therefore be discarded.

Similarly, consider terms linear in the external fields. As far as $H_{\mathrm{FW}}$ itself is concerned, only two factors of $p$ need to be taken into account; the rightmost of them generates a 
second external field when applied to the zero-velocity state, and this external field can absorb the other factor of $p$ as a derivative. However, any additional factor $p$ would commute through to the right and generate a third external field when applied to the zero-velocity state. Again, however, one has to also ensure that sufficiently many terms in $G_{n}$ are kept. Keeping three powers of $p$ in $H_{\mathrm{FW}}$ corresponds to having two powers of $p$ in $G_{n}$; the rightmost one generates a second external field, which can absorb the other factor of $p$ as a derivative, rendering $G_{n}$ simply quadratic in the external fields with no further factors of $p$. However, if there were yet one more factor of $p$, then it would commute through to the right and generate a third external field.

Finally, consider the overall order in $E, B, \partial / \partial t, \nabla, p$. Certainly, as far as $H_{\mathrm{FW}}$ itself is concerned, altogether fourth order is sufficient: Every factor $p$ generates either an external field or a derivative; the desired limitation to at most two external fields, which each can absorb at most one derivative, implies that only terms up to fourth order in these objects collectively are relevant. Now, keeping terms up to fourth order in $H_{\mathrm{FW}}$ corresponds to consistently keeping only terms up to third order in $G_{n}$. This is nevertheless sufficient, because in $H_{\mathrm{FW}}$, any rightmost factor $p$ always comes multiplied with external fields or further factors of $p$. Thus, after applying said rightmost factor $p$ to the zero-velocity state, generating a factor $G_{n}$, that factor $G_{n}$ always comes multiplied with another factor of $p$ or an external field. Therefore, the fourth order terms in $G_{n}$ are in fact irrelevant and it is indeed sufficient to construct $G_{n}$ consistently only to third order. Thus, in $H_{\mathrm{FW}}$ only altogether fourth order in $E$, $B, \partial / \partial t, \nabla, p$ is required.

Continuing with the above scheme of eliminating the momentum operator to derive the action of $H_{\mathrm{FW}}$ on a zerovelocity state, note that the identification (52) in general introduces an ambiguity: Since the operators $p_{i}$ and $G_{j}$ do not commute, the order in which one applies momentum operators can make a difference, i.e., in general it can happen that, effectively, $\left[p_{i}, p_{j}\right] \neq 0$. However, zero-velocity states on which this occurs presumably should not be viewed as acceptable solutions of the present small-field perturbative expansion. If $\left[p_{i}, p_{j}\right] \neq 0$ when applied to a wave function, this implies that the wave function contains singular vorticity. It thus differs strongly from zero-momentum states, which are spatially constant. However, the present smallfield expansion presupposes that zero-velocity states are perturbations of zero-momentum states, with corrections suppressed by powers of the small external fields. For zero external field, zero momentum and zero velocity coincide. Thus, states with singular vorticity that yield $\left[p_{i}, p_{j}\right] \neq 0$ ought to be excluded from consideration a priori in order to preserve a consistent perturbative small-field expansion scheme. Thus, the requirements for a consistent treatment would appear to include treating products of momentum operators as

$$
p_{i} p_{j}=\frac{1}{2}\left\{p_{i}, p_{j}\right\}+\frac{1}{2}\left[p_{i}, p_{j}\right]
$$

and setting $\left[p_{i}, p_{j}\right]=0$. This working assumption will be explored further below and a concluding critique will be given in Sec. IV D.

To exhibit clearly the emergence of the ambiguities discussed above, it is useful to proceed as follows. Consider, to begin with, the operator $p^{2}=p_{n} p_{n}$ appearing in $H_{\mathrm{FW}}$. In this operator, no ordering ambiguity arises regarding an initial application of the identification (52). Multiplying (52) from the left with $p_{n}$ and commuting all momentum operators through to the right yields (as above, in the following, $\nabla \cdot B=0$ will be dropped by virtue of Maxwell's equations for the external fields)

$$
\begin{aligned}
p^{2}= & i \mu \sigma \cdot(\nabla \times E)+\mu \sigma \cdot(E \times p)+\frac{\mu^{2}}{2 m^{2}}\left(-3 \nabla_{i} E_{j} \nabla_{i} E_{j}+\nabla_{i} E_{j} \nabla_{j} E_{i}+i \sigma \cdot(\nabla \times E) \nabla \cdot E+(\nabla \cdot E)^{2}+\nabla_{i} B_{j} \nabla_{j} B_{i}\right) \\
& +\frac{\mu}{2 m^{2}}\left(2 i \epsilon_{i j k} \sigma_{k} \nabla_{l} E_{i} p_{l} p_{j}-i \sigma \cdot(\nabla \times E) p^{2}-\nabla \cdot E p^{2}\right)+\frac{\mu}{4 m}\left(\dot{E} \cdot p-(\nabla \times B) \cdot p+3 i \sigma_{i} \nabla_{i} B_{j} p_{j}-4 \sigma_{j} B_{i} p_{j} p_{i}\right) .
\end{aligned}
$$

In this form, one now observes potential ordering ambiguities in the third and fourth lines. However, instead of contemplating further manipulations of this form on its own, one can proceed by inserting it into the full Hamiltonian $H_{\mathrm{FW}}$, upon which one observes several cancellations of these potential ambiguities,

$$
\begin{aligned}
H_{\mathrm{FW}}= & m-\mu \sigma \cdot B-\frac{\mu}{2 m} \nabla \cdot E+\frac{\mu^{2}}{2 m} E^{2}+\frac{\mu^{2}}{8 m^{2}}\left(2 \sigma \cdot(E \times \dot{E})-3 \sigma \cdot(E \times(\nabla \times B))-\sigma_{i} E_{j}\left(\nabla_{i} B_{j}+\nabla_{j} B_{i}\right)\right. \\
& \left.-2 \sigma_{i} B_{j}\left(\nabla_{i} E_{j}+\nabla_{j} E_{i}\right)\right)+\frac{\mu^{2}}{32 m^{3}}\left(4 \dot{E}^{2}-8(\nabla \times B) \cdot \dot{E}+2(\nabla \times B)^{2}+\left(\nabla_{i} B_{j}+\nabla_{j} B_{i}\right)^{2}-12 \nabla_{i} E_{j} \nabla_{i} E_{j}\right. \\
& \left.+4 \nabla_{i} E_{j} \nabla_{j} E_{i}+4(\nabla \cdot E)^{2}\right)-\frac{\mu}{2 m} \sigma \cdot(E \times p)+\frac{\mu}{8 m^{2}}\left((\nabla \times B) \cdot p-\dot{E} \cdot p+i \sigma_{i} \nabla_{i} B_{j} p_{j}\right)-\frac{1}{8 m^{3}} p^{4} .
\end{aligned}
$$


Fortuitously, only one ambiguous term remains, namely the term proportional to $p^{4}$, which will be considered separately below. Aside from that term, there are only ones containing at most one power of momentum, and therefore no ordering ambiguities. Since the terms containing one power of momentum already exhibit one power of the external fields, only a few of the terms appearing in (52) in conjunction with (50) remain relevant when eliminating that momentum operator; the other terms only contribute at the third order in the external fields or higher. Namely, only the first four terms in (50) must be retained, and also the $p^{2}$ operator in the parentheses in (52) can be dropped. Carrying out this elimination yields

$$
\begin{aligned}
H_{\mathrm{FW}}= & m-\mu \sigma \cdot B-\frac{\mu}{2 m} \nabla \cdot E-\frac{\mu^{2}}{2 m} E^{2}+\frac{\mu^{2}}{64 m^{3}}\left(6 \dot{E}^{2}-3(\nabla \times B)^{2}-12(\nabla \times B) \cdot \dot{E}\right)+\frac{7 \mu^{2}}{128 m^{3}}\left(\nabla_{i} B_{j}+\nabla_{j} B_{i}\right)^{2} \\
& +\frac{\mu^{2}}{8 m^{3}}\left((\nabla \cdot E)^{2}-3 \nabla_{i} E_{j} \nabla_{i} E_{j}+\nabla_{i} E_{j} \nabla_{j} E_{i}\right)-\frac{\mu^{2}}{4 m^{2}}\left(\sigma \cdot(E \times(\nabla \times B))+\sigma_{i} B_{j}\left(\nabla_{i} E_{j}+\nabla_{j} E_{i}\right)\right)-\frac{1}{8 m^{3}} p^{4} .
\end{aligned}
$$

It remains to treat the operator $p^{4}$. Multiplying the form (54) for the operator $p^{2}$ by another factor $p^{2}$ from the left, one may discard the majority of the terms because they only contribute at orders that are dropped in the expansion pursued here. The remaining relevant terms are

$$
p^{4}=p^{2}[i \mu \sigma \cdot(\nabla \times E)+\mu \sigma \cdot(E \times p)] .
$$

Two ways of proceeding suggest themselves: On the one hand, one may view $p^{4}$ as the successive application of two $p^{2}$ operators, i.e., first fully resolve the term in the square brackets by applying the remaining momentum operator to the zero-velocity state; on the other hand, one may follow the general scheme laid out above, commuting all momentum operators to the right in (57) immediately, and then treating them in a symmetrized fashion as suggested by (53). Note that the fully symmetrized form $p^{4}=(1 / 3)\left(p_{i} p_{i} p_{j} p_{j}+\right.$ $\left.p_{i} p_{j} p_{i} p_{j}+p_{i} p_{j} p_{j} p_{i}\right)$ corresponds to a weighted average of these two alternatives.

Starting with the former procedure, by inserting (52) and only keeping relevant terms, one obtains

$$
\begin{gathered}
p^{4}=p^{2}\left[i \mu \sigma \cdot(\nabla \times E)+\mu^{2} \sigma \cdot(E \times(\sigma \times E))\right] \\
=p^{2}\left[i \mu \sigma \cdot(\nabla \times E)+2 \mu^{2} E^{2}\right] \\
=i \mu \sigma \cdot(\nabla \times E) p^{2}-4 \mu^{2} \nabla_{i} E_{j} \nabla_{i} E_{j} \\
=[i \mu \sigma \cdot(\nabla \times E)]^{2}-4 \mu^{2} \nabla_{i} E_{j} \nabla_{i} E_{j} \\
=-5 \mu^{2} \nabla_{i} E_{j} \nabla_{i} E_{j}+\mu^{2} \nabla_{i} E_{j} \nabla_{j} E_{i} .
\end{gathered}
$$

On the other hand, immediately commuting momentum operators to the right in (57), one has

$$
p^{4}=i \mu \sigma \cdot(\nabla \times E) p^{2}-i \mu \epsilon_{i j k} \sigma_{i} \nabla_{l} E_{j}\left\{p_{l}, p_{k}\right\}
$$

exhibiting the ambiguity in ordering momentum operators in the last term, treated as suggested by (53). Again inserting (52) and only keeping relevant terms, one has

$$
p^{4}=\mu^{2}(\nabla \cdot E)^{2}-4 \mu^{2} \nabla_{i} E_{j} \nabla_{i} E_{j}+\mu^{2} \nabla_{i} E_{j} \nabla_{j} E_{i}
$$

having used $(\nabla \times E)_{j}\left(\nabla_{i} E_{j}-\nabla_{j} E_{i}\right)=0$. The expressions (62) and (64) disagree; evidently, varying the ordering of momentum operators corresponds to trading off terms $(\nabla \cdot E)^{2}$ and $-\nabla_{i} E_{j} \nabla_{i} E_{j}$ in the Foldy-Wouthuysen Hamiltonian. The coefficients of these terms in $H_{\mathrm{FW}}$ are ambiguous unless a cogent rationale for choosing a particular ordering can be constructed. This will be revisited in Sec. IV D. The term $\nabla_{i} E_{j} \nabla_{j} E_{i}$ does appear to be determined with a unique coefficient, such that the $\nabla_{i} E_{j} \nabla_{j} E_{i}$ contribution completely cancels in $H_{\mathrm{FW}}$. With the ambiguous terms unresolved, the energy of the neutron in a zerovelocity state $|v=0\rangle$ takes the form

$$
\begin{aligned}
W_{\mathrm{FW}}^{v=0}= & m-\mu \sigma \cdot B-\frac{\mu}{2 m} \nabla \cdot E-\frac{\mu^{2}}{2 m} E^{2} \\
& +\frac{\mu^{2}}{64 m^{3}}\left[6 \dot{E}^{2}-3(\nabla \times B)^{2}-12(\nabla \times B) \cdot \dot{E}\right] \\
& +\frac{7 \mu^{2}}{128 m^{3}}\left(\nabla_{i} B_{j}+\nabla_{j} B_{i}\right)^{2}-\frac{\mu^{2}}{4 m^{2}}(\sigma \cdot(E \times(\nabla \times B)) \\
& \left.+\sigma_{i} B_{j}\left(\nabla_{i} E_{j}+\nabla_{j} E_{i}\right)\right)+O\left((\nabla \cdot E)^{2}\right)+O\left(\nabla_{i} E_{j} \nabla_{i} E_{j}\right) .
\end{aligned}
$$

Specializing to the vacuum and using the vacuum Maxwell equations for the external fields, which allows one to combine the terms grouped in the square brackets, one arrives at

$$
\begin{aligned}
W_{\mathrm{FW}}^{v=0}= & m-\mu \sigma \cdot B-\frac{\mu^{2}}{2 m} E^{2}-\frac{9 \mu^{2}}{64 m^{3}} \dot{E}^{2} \\
& +\frac{7 \mu^{2}}{128 m^{3}}\left(\nabla_{i} B_{j}+\nabla_{j} B_{i}\right)^{2}-\frac{\mu^{2}}{4 m^{2}} \sigma \cdot(E \times \dot{E}) \\
& -\frac{\mu^{2}}{4 m^{2}} \sigma_{i} B_{j}\left(\nabla_{i} E_{j}+\nabla_{j} E_{i}\right) \\
& +O\left(\left(\nabla_{i} E_{j}+\nabla_{j} E_{i}\right)^{2}\right)+O\left(\dot{B}^{2}\right)
\end{aligned}
$$


taking into account the decomposition $\nabla_{i} E_{j} \nabla_{i} E_{j}=$ $(1 / 4)\left[\left(\nabla_{i} E_{j}+\nabla_{j} E_{i}\right)^{2}+\left(\nabla_{i} E_{j}-\nabla_{j} E_{i}\right)^{2}\right]$ as well as $\left(\nabla_{i} E_{j}-\nabla_{j} E_{i}\right)^{2}=2(\nabla \times E)^{2}=2 \dot{B}^{2}$. Compared to the $p=0$ case, the $E^{2}$ term changes sign, as has been previously observed in [55]. Also the higher order terms are modified substantially, some disappearing entirely and new ones appearing. Two terms remain undetermined in the $v=0$ case.

\section{Action of Foldy-Wouthuysen Hamiltonian on a $\left|v^{2}=0\right\rangle$ state}

Before considering the relative merits of the different schemes of treating the ordering of momentum operators exhibited in the previous section, it is useful to also have at hand the expressions resulting when acting on a $\left|v^{2}=0\right\rangle$ state, for which only the property $v^{2}\left|v^{2}=0\right\rangle=0$ is assumed. Proceeding in analogy to the argument leading to Eq. (52), taking the square of Eq. (49) yields

$$
v^{2}=\frac{p^{2}}{m^{2}}\left(1-\frac{p^{2}}{m^{2}}\right)+F
$$

with $F$ summarizing all the terms that are at least linear in the external fields,

$$
F=\frac{p_{n}}{m}\left(1-\frac{p^{2}}{2 m^{2}}\right) G_{n}+G_{n} \frac{p_{n}}{m}\left(1-\frac{p^{2}}{2 m^{2}}\right)+G_{n} G_{n} .
$$

This can be rearranged to construct an iterative scheme for eliminating $p^{2}$ in favor of $v^{2}$,

$$
\begin{aligned}
p^{2} & =m^{2}\left(1-\frac{p^{2}}{m^{2}}\right)^{-1}\left(v^{2}-F\right) \\
& =m^{2}\left(1+\frac{p^{2}}{m^{2}}+\cdots\right)\left(v^{2}-F\right)
\end{aligned}
$$

and thus, applied to a $\left|v^{2}=0\right\rangle$ state,

$$
\begin{gathered}
p^{2}=-\left(m^{2}+p^{2}\right) F \\
=-m p_{n}\left(1+\frac{p^{2}}{2 m^{2}}\right) G_{n}-m\left(1+\frac{p^{2}}{m^{2}}\right) G_{n} p_{n} \\
-\left(m^{2}+p^{2}\right) G_{n} G_{n} .
\end{gathered}
$$

Note that the first term in (71) corresponds to Eq. (52) with an extra $p_{n}$ applied from the left; i.e., the expression for $p^{2}$ obtained here, acting on a $\left|v^{2}=0\right\rangle$ state, differs from the one obtained when acting on a $|v=0\rangle$ state, cf. (54), by the two additional terms in (71). For the discussion in the next section, it is useful to observe that these additional terms can also be cast as follows:

$$
p^{2}=-m p_{n}\left(1+\frac{p^{2}}{2 m^{2}}\right) G_{n}-\left(m^{2}+p^{2}\right) G_{n} v_{n}
$$

as can be verified by inserting (49), again discarding terms that are of too high order. Note that any $|v=0\rangle$ state is also a $\left|v^{2}=0\right\rangle$ state, and therefore the expressions for $p^{2}$ derived in the two cases must be consistent with one another when applied to $|v=0\rangle$ states. Indeed, the additional terms vanish when one uses $v_{n}|v=0\rangle=0$. On the other hand, for $\left|v^{2}=0\right\rangle$ states that are not also known to be $|v=0\rangle$ states, there is no a priori guarantee that the additional terms vanish.

To assemble the Foldy-Wouthuysen Hamiltonian acting on $\left|v^{2}=0\right\rangle$ states, one can reuse the result (55), merely supplementing it with the additional terms entering $p^{2}$ [multiplied by the appropriate prefactor, $1 /(2 m)$ ]. Using (50), commuting momentum operators to the right, and dropping terms that are of too high order, these terms read

$$
\begin{aligned}
-m G_{n} p_{n}= & \mu(\sigma \times E) \cdot p \\
& -\frac{\mu}{4 m}\left(-\dot{E} \cdot p+(\nabla \times B) \cdot p-i \sigma_{i} \nabla_{i} B_{n} p_{n}\right) \\
& -\frac{\mu}{2 m^{2}}\left(-2 i \sigma_{j} \epsilon_{j i n} \nabla_{k} E_{i} p_{k} p_{n}+i \sigma \cdot(\nabla \times E) p^{2}\right. \\
& \left.+(\nabla \cdot E) p^{2}\right)-\frac{\mu}{m} \sigma_{i} B_{n} p_{i} p_{n}
\end{aligned}
$$

$$
-\frac{p^{2}}{m} G_{n} p_{n}=-2 i \frac{\mu}{m^{2}} \epsilon_{i j k} \sigma_{j} \nabla_{l} E_{k} p_{l} p_{i}
$$

$$
\begin{aligned}
-m^{2} G_{n} G_{n}= & -m^{2}\left(-\frac{\mu}{m} \sigma \times E+\frac{\mu}{4 m^{2}}\left(-\dot{E}+\nabla \times B-i \sigma_{i} \nabla_{i} B\right)\right)^{2}+\frac{\mu^{2}}{2 m^{2}}\left(-4 \nabla_{i} E_{j} \nabla_{i} E_{j}+\nabla_{i} E_{j} \nabla_{j} E_{i}\right. \\
& \left.+(\nabla \cdot E)^{2}+(\nabla \cdot E) i \sigma \cdot(\nabla \times E)\right)+\frac{\mu^{2}}{2 m}\left(3 \sigma_{j} B_{i} \nabla_{i} E_{j}-(\nabla \cdot E)(\sigma \cdot B)-i B \cdot(\nabla \times E)\right)
\end{aligned}
$$

In addition, in the operator $p^{4}=p^{2} p^{2}$, one can likewise substitute the right-hand $p^{2}$ with (71); after commuting the 
other $p^{2}$ operator to the right and discarding terms of too high order, the remaining terms are

$$
\begin{aligned}
p^{4}= & i \mu \sigma \cdot(\nabla \times E) p^{2}-4 i \mu \epsilon_{i j k} \sigma_{i} \nabla_{l} E_{j} p_{k} p_{l} \\
& +4 \mu^{2} \nabla_{i} E_{j} \nabla_{i} E_{j} .
\end{aligned}
$$

Consider, to begin with, the terms now appearing in $H_{\mathrm{FW}}$ that are linear in $B$ and contain no $E$. These read

$$
\begin{aligned}
\left.H_{\mathrm{FW}}\right|_{E=0, \text { linearin } B}= & -\mu \sigma \cdot B \\
& +\frac{\mu}{4 m^{2}}\left(i \sigma_{i} \nabla_{i} B_{j} p_{j}-2 \sigma_{i} B_{j} p_{i} p_{j}\right) .
\end{aligned}
$$

This form implies that one reaches an impasse in the treatment of external magnetic fields; the present scheme of acting on a $\left|v^{2}=0\right\rangle$ state only allows one to eliminate the operator $p^{2}$, but not individual components $p_{n}$. The above terms thus cannot be evaluated any further. The $\left|v^{2}=0\right\rangle$ state scheme therefore has the significant drawback of not permitting a well-defined treatment of external magnetic fields. In the following, only the case $B=0$ will therefore be considered.

Fortuitously, when one assembles the terms containing electric fields, all problematic terms cancel and one is left at most with additional factors $p^{2}$ to resolve. Note that, when already multiplied by one power of an external field from the left, the only term in $p^{2}$ that remains relevant to the desired order is $i \mu \sigma \cdot(\nabla \times E)$. Carrying out the remaining evaluation, one arrives at the Foldy-Wouthuysen Hamiltonian:

$$
\begin{aligned}
\left.H_{\mathrm{FW}}\right|_{B=0}= & m-\frac{\mu}{2 m} \nabla \cdot E-\frac{\mu^{2}}{2 m} E^{2}+\frac{3 \mu^{2}}{32 m^{3}} \dot{E}^{2} \\
& +\frac{3 \mu^{2}}{8 m^{3}}(\nabla \cdot E)^{2}+\frac{\mu^{2}}{2 m^{3}} \nabla_{i} E_{j} \nabla_{i} E_{j} .
\end{aligned}
$$

This reproduces the form obtained for $|v=0\rangle$ states, except for the ambiguous $(\nabla \cdot E)^{2}$ and $\nabla_{i} E_{j} \nabla_{i} E_{j}$ terms, which appear here with yet different coefficients than in either of the alternative schemes discussed in the previous section.

\section{Inconsistencies in defining a $|v=0\rangle$ state at order $\boldsymbol{O}(\boldsymbol{\nabla} E \nabla E)$}

As has been argued already further above, a consistent zero-velocity state $|v=0\rangle$ ought to satisfy $\left[p_{i}, p_{j}\right] \mid v=$ $0\rangle=0$ in order to represent a bona fide small-field perturbation of a zero-momentum state. This condition was used to resolve the ordering ambiguity in products of momentum operators, cf. (53); whenever a product of momentum operators acts on a zero-velocity state, the product is to be symmetrized.

However, this prescription cannot be consistently maintained under all circumstances. Recalling the discussion of Eqs. (71) and (72), since any $|v=0\rangle$ state is also a $\left|v^{2}=0\right\rangle$ state, the expression (71) for the operator $p^{2}$ acting on a $\left|v^{2}=0\right\rangle$ state must equal the expression obtained when acting on a $|v=0\rangle$ state, which only includes the first term in (71). Indeed, as exhibited in Eq. (72), the additional terms vanish on $|v=0\rangle$ states, since they can be written in terms of an operator that includes a factor $v_{n}$ on the right. This, however, supposes a definite ordering of operators that is inconsistent with a symmetrization prescription: In the product

$$
G_{n} v_{n}=G_{n}\left(\frac{p_{n}}{m}+G_{n}\right)
$$

the operator $p_{n}$ must be kept to the right of any momentum operators appearing in the $G_{n}$ outside of the parentheses. If one instead were to symmetrize the product of $p_{n}$ with momentum operators occurring to its left, one would alter the product $G_{n} v_{n}$, which vanishes when acting on $|v=0\rangle$ states, into a different operator $\Gamma$ that is not anymore a product of two factors $G_{n}$ and $v_{n}$, but represents a new composite operator that does not vanish when acting on $|v=0\rangle$ states. One could, in effect, come to the absurd conclusion that

$$
\begin{aligned}
0 & =G_{n} v_{n}|v=0\rangle=G_{n}\left(\frac{p_{n}}{m}+G_{n}\right)|v=0\rangle \\
& =: \Gamma|v=0\rangle \neq 0,
\end{aligned}
$$

where the nonvanishing terms again involve $(\nabla \cdot E)^{2}$ and $\nabla_{i} E_{j} \nabla_{i} E_{j}$. It appears, therefore, that, once one attempts to determine its energy to an accuracy including the order $O(\nabla E \nabla E)$, there exists no $|v=0\rangle$ state consistently defined to that accuracy. One may speculate that this is a signature of a conflict between the necessarily extended nature of a zero-velocity state in space and the limited spatial range of a description in terms of a derivative expansion. Ultimately, it does not appear feasible within the present framework to determine unambiguously contributions to the energy of a neutron at rest that are proportional to $(\nabla \cdot E)^{2}$ and $\nabla_{i} E_{j} \nabla_{i} E_{j}$. It remains unclear whether retreating to a calculational scheme based on $\left|v^{2}=0\right\rangle$ states can provide a resolution of the ambiguities associated with these terms, or whether it merely hides them through its relative inflexibility in exploring different operator orderings. In any case, the $\left|v^{2}=0\right\rangle$ scheme does not allow one to treat magnetic background fields, as seen in the previous section.

\section{CONCLUSIONS}

By constructing an appropriate Foldy-Wouthuysen transformation, the energy of a pointlike neutron in an external electromagnetic field was determined in a combined expansion in powers of the external field and derivatives thereof. Both the case of a zero-momentum state as well as the case of a zero-velocity state were 
considered, leading to the results (41) and (66), respectively, in the absence of external charges or currents. The obtained terms mirror the ones appearing in the effective Hamiltonian (1), mimicking the effects of the polarizabilities defined there. This generalizes the long-known result of Foldy [54] pertaining specifically to the dipole electric polarizability $\alpha_{E}$. In order to separate the dipole electric polarizability proper, i.e., the effect of an actual structural deformation of an extended neutron, from the energy shift already experienced by a pointlike neutron, the contribution $\alpha_{E}^{\text {Foldy }}=-\mu^{2} / m$ (in a zero-momentum state) must be subtracted from the coefficient $\alpha_{E}$ found in the effective Hamiltonian (1). Comparing the zeromomentum result (41) with (1), the ten Foldy contributions for a zero-momentum neutron are

$$
\begin{aligned}
& \alpha_{E}^{\text {Foldy }, p=0}=-\frac{\mu^{2}}{m}, \quad \beta_{M}^{\text {Foldy }, p=0}=0, \quad \alpha_{E \nu}^{\text {Foldy }, p=0}=0, \\
& \beta_{M \nu}^{\text {Foldy }, p=0}=-\frac{\mu^{2}}{2 m^{3}}, \quad \alpha_{E 2}^{\text {Foldy }, p=0}=-\frac{3 \mu^{2}}{m^{3}}, \quad \beta_{M 2}^{\text {Foldy }, p=0}=0, \\
& \gamma_{E 1}^{\text {Foldy }, p=0}=\frac{\mu^{2}}{4 m^{2}}, \quad \gamma_{M 1}^{\text {Foldy }, p=0}=0, \quad \gamma_{E 2}^{\text {Foldy }, p=0}=-\frac{\mu^{2}}{2 m^{2}}, \\
& \gamma_{M 2}^{\text {Foldy }, p=0}=\frac{\mu^{2}}{4 m^{2}} .
\end{aligned}
$$

On the other hand, eliminating the momentum operator in favor of the velocity operator, one can construct analogous contributions in the zero-velocity case. In the course of this construction, operator-ordering ambiguities were encountered that appear to signal an inconsistency in defining a zero-velocity state to order $O(\nabla E \nabla E)$. This is presumably due to a conflict between the spatially extended nature of such a state and the limitation of a derivative expansion in capturing the associated spatial behavior. The ambiguities specifically arise in the treatment of the $p^{4}$ term representing a relativistic correction to the kinetic energy. They are, therefore, a relativistic effect, but understanding these relativistic effects is evidently necessary for a proper treatment of $O(\nabla E \nabla E)$ terms. These ambiguities precluded a determination of the Foldy contributions for $\alpha_{E 2}$ and $\beta_{M \nu}$. It should be emphasized that, in view of the exhaustive treatment presented in Sec. IV of the consequences of assuming the existence of a zero-velocity state, the ambiguities appear to be a consequence already of the initial assumption of the neutron energy being represented by an expansion of the form (1). To remove them would require abandoning the form (1) rather than merely improving upon the calculational scheme employed here. The study of forms more general than (1) lies beyond the scope of this work. Comparing the result (66) with (1), the remaining eight Foldy contributions for a zero-velocity neutron are

$$
\begin{aligned}
& \alpha_{E}^{\text {Foldy }, v=0}=\frac{\mu^{2}}{m}, \quad \beta_{M}^{\text {Foldy }, v=0}=0, \quad \alpha_{E \nu}^{\text {Foldy }, v=0}=\frac{9 \mu^{2}}{32 m^{3}} \\
& \beta_{M 2}^{\text {Foldy }, v=0}=-\frac{21 \mu^{2}}{8 m^{3}}, \quad \gamma_{E 1}^{\text {Foldy }, v=0}=\frac{\mu^{2}}{2 m^{2}}, \quad \gamma_{M 1}^{\text {Foldy }, v=0}=0 \\
& \gamma_{E 2}^{\text {Foldy }, v=0}=-\frac{\mu^{2}}{2 m^{2}}, \quad \gamma_{M 2}^{\text {Foldy }, v=0}=0 .
\end{aligned}
$$

As already noted in [55], the sign of the Foldy contribution for $\alpha_{E}$ is inverted going from the zero-momentum to the zero-velocity case. Also the majority of the other contributions changes.

An application of these results that suggests itself is the analysis of lattice QCD calculations of hadron mass shifts in the presence of external electromagnetic fields. Which of the scenarios investigated here is relevant for such an analysis depends sensitively on the details of the lattice setup; there is no simple, unique prescription for disentangling the mass shift due to the genuine polarization of the hadron's internal structure from the Foldy-type contributions discussed here, additional perturbative effects such as the ones exhibited in Sec. III B, and a variety of other systematic corrections.

The calculational scheme employed in $[28,29,32]$ preserves spatial translational invariance and thereby is able to place the neutron in a zero-momentum state. To correct for Foldy-type effects, the $p=0$ results (82) are therefore relevant; in addition, the perturbative corrections discussed in Sec. III B apply. It should be noted that this relative simplicity of the spatial setup comes at the price of using explicitly time-dependent external electromagnetic gauge fields that induce a complicated nonstationary time evolution; the extraction of the neutron mass shift requires a delicate analysis of the resulting transitory neutron state.

In order to avoid such complications, the scheme employed in [33-37,39] instead uses Dirichlet boundary conditions for the valence quarks in some space-time directions, which permits one to introduce arbitrarily weak, temporally constant external gauge fields. However, one consequence of using Dirichlet boundary conditions is that the hadron cannot be in a zero-momentum state, but instead acquires a nontrivial spatial wave function with typical momenta of the order of $\pi / L$ (where $L$ denotes the spatial extent of the lattice), including also distortions of the hadron's structure through interactions with the hard walls. Analysis of the resulting data requires a detailed study of the dependence on $L$. In this setup, the expectation value of the hadron velocity vanishes for some directions $i,\left\langle v_{i}\right\rangle=0$. Keeping in mind the caveat already raised in Sec. IVA, that $\langle v\rangle=0$ is a considerably weaker condition than the presence of a true $|v=0\rangle$ or $\left|v^{2}=0\right\rangle$ state, the resulting hadron wave functions have the character of anisotropic, hybrid zero-velocity/zero-momentum states. While the $v=0$ results (83) are thus not directly applicable, the developments in Sec. IV provide a calculational scheme that can be 
adapted to treat such more specialized scenarios, together with an indication of their possible limitations.

More complicated spatial structures are induced for charged particles in a magnetic background field, which are described by Landau levels in the plane perpendicular to the magnetic field. Significant effort is required to achieve a good overlap with these states in lattice calculations, through an $S U(3) \times U(1)$ eigenmode projection technique 42]], along with a hadronic Landau eigenmode projection to the lowest Landau level, to extract polarizabilities for charged pions and the proton [43-45]. Due to the localization of the Landau levels in the directions perpendicular to the magnetic field (whereas propagation along the direction of the field is free), the charged hadron wave functions again have the character of hybrid zero-velocity/ zero-momentum states, and the analysis of possible Foldytype effects would require further, more specific study that lies beyond the scope of the present work.

In view of the manifold details characterizing any particular lattice setup, no exhaustive conclusions can be drawn concerning the accounting for Foldy-type effects in lattice QCD polarizability calculations. Nonetheless, we anticipate the two scenarios studied here to be relevant for the analysis of selected computations, a case in point being further developments within the zero-momentum scheme developed to study the dipole [28,29] and spin polarizabilities [32].

\section{ACKNOWLEDGMENTS}

Fruitful discussions with S. A. Coon, W. Detmold, H. Grießhammer and B. Pasquini are acknowledged. This research was supported by the Erwin Schrödinger Fellowship program of the Austrian Science Fund FWF ("Fonds zur Förderung der wissenschaftlichen Forschung") under Contract No. J3425-N27 (R. H.) and the U.S. Department of Energy, Office of Science, Office of Nuclear Physics through Grant No. DE-FG02-96E R40965 (M. E. and J. S.).
[1] D. Babusci, G. Giordano, A. I. L'vov, G. Matone, and A. M. Nathan, Phys. Rev. C 58, 1013 (1998).

[2] B. Holstein, D. Drechsel, B. Pasquini, and M. Vanderhaeghen, Phys. Rev. C 61, 034316 (2000).

[3] D. Drechsel, B. Pasquini, and M. Vanderhaeghen, Phys. Rep. 378, 99 (2003).

[4] R. Hildebrandt, H. Grießhammer, T. Hemmert, and B. Pasquini, Eur. Phys. J. A 20, 293 (2004).

[5] M. Schumacher, Prog. Part. Nucl. Phys. 55, 567 (2005).

[6] B. Pasquini, D. Drechsel, and M. Vanderhaeghen, Phys. Rev. C 76, 015203 (2007).

[7] B. Pasquini, P. Pedroni, and D. Drechsel, Phys. Lett. B 687, 160 (2010).

[8] H. Grießhammer, J. McGovern, D. R. Phillips, and G. Feldman, Prog. Part. Nucl. Phys. 67, 841 (2012).

[9] J. McGovern, D. R. Phillips, and H. Grießhammer, Eur. Phys. J. A 49, 12 (2013).

[10] B. Holstein and S. Scherer, Annu. Rev. Nucl. Part. Sci. 64, 51 (2014).

[11] L. S. Myers et al. (COMPTON@MAX-lab Collaboration), Phys. Rev. Lett. 113, 262506 (2014).

[12] P. Martel et al. (A2 Collaboration), Phys. Rev. Lett. 114, 112501 (2015).

[13] O. Gryniuk, F. Hagelstein, and V. Pascalutsa, Phys. Rev. D 92, 074031 (2015).

[14] O. Gryniuk, F. Hagelstein, and V. Pascalutsa, Phys. Rev. D 94, 034043 (2016).

[15] F. Hagelstein, R. Miskimen, and V. Pascalutsa, Prog. Part. Nucl. Phys. 88, 29 (2016).

[16] H. Grießhammer, J. McGovern, and D. R. Phillips, Eur. Phys. J. A 54, 37 (2018).
[17] B. Pasquini, P. Pedroni, and S. Sconfietti, Phys. Rev. C 98, 015204 (2018).

[18] B. Pasquini and M. Vanderhaeghen, Annu. Rev. Nucl. Part. Sci. 68, 75 (2018).

[19] B. Pasquini, P. Pedroni, and S. Sconfietti, J. Phys. G 46, 104001 (2019).

[20] R. Miskimen, Proc. Sci., CD2018 (2019) 015.

[21] P. Martel et al. (A2 Collaboration), Proc. Sci., CD2018 (2019) 038 .

[22] D. Paudyal et al. (A2 Collaboration), Phys. Rev. C 102, 035205 (2020).

[23] J. Melendez, R. Furnstahl, H. Grießhammer, J. McGovern, D. R. Phillips, and M. Pratola, Eur. Phys. J. A 57, 81 (2021).

[24] H. Fiebig, W. Wilcox, and R. Woloshyn, Nucl. Phys. B324, 47 (1989).

[25] J. Christensen, W. Wilcox, F. X. Lee, and L. Zhou, Phys. Rev. D 72, 034503 (2005).

[26] F. X. Lee, L. Zhou, W. Wilcox, and J. Christensen, Phys. Rev. D 73, 034503 (2006).

[27] E. Shintani, S. Aoki, N. Ishizuka, K. Kanaya, Y. Kikukawa, Y. Kuramashi, M. Okawa, A. Ukawa, and T. Yoshie, Phys. Rev. D 75, 034507 (2007).

[28] M. Engelhardt, Phys. Rev. D 76, 114502 (2007).

[29] M. Engelhardt, Proc. Sci., LAT2009 (2009) 128.

[30] W. Detmold, B. Tiburzi, and A. Walker-Loud, Phys. Rev. D 79, 094505 (2009).

[31] W. Detmold, B. Tiburzi, and A. Walker-Loud, Phys. Rev. D 81, 054502 (2010).

[32] M. Engelhardt, Proc. Sci., Lattice 2011 (2011) 153.

[33] A. Alexandru and F. X. Lee, Proc. Sci., LAT2009 (2009) 144. 
[34] F. X. Lee and A. Alexandru, Proc. Sci., Lattice 2010 (2010) 148.

[35] F. X. Lee and A. Alexandru, Proc. Sci., Lattice 2011 (2011) 317.

[36] M. Lujan, A. Alexandru, W. Freeman, and F. X. Lee, Phys. Rev. D 89, 074506 (2014).

[37] W. Freeman, A. Alexandru, M. Lujan, and F. X. Lee, Phys. Rev. D 90, 054507 (2014).

[38] E. V. Luschevskaya, O. E. Solovjeva, O. A. Kochetkov, and O. V. Teryaev, Nucl. Phys. B898, 627 (2015).

[39] M. Lujan, A. Alexandru, W. Freeman, and F. X. Lee, Phys. Rev. D 94, 074506 (2016).

[40] T. Primer, W. Kamleh, D. Leinweber, and M. Burkardt, Phys. Rev. D 89, 034508 (2014).

[41] R. Bignell, J. Hall, W. Kamleh, D. Leinweber, and M. Burkardt, Phys. Rev. D 98, 034504 (2018).

[42] R. Bignell, W. Kamleh, and D. Leinweber, Phys. Rev. D 101, 094502 (2020).

[43] R. Bignell, W. Kamleh, and D. Leinweber, Phys. Lett. B 811, 135853 (2020).

[44] F. He, D. Leinweber, A. Thomas, and P. Wang, Phys. Rev. D 102, 114509 (2020).

[45] R. Bignell, W. Kamleh, and D. Leinweber, EPJ Web Conf. 245, 06033 (2020).
[46] W. Detmold, B. Tiburzi, and A. Walker-Loud, Phys. Rev. D 73, 114505 (2006).

[47] V. Lensky, J. McGovern, and V. Pascalutsa, Eur. Phys. J. C 75, 604 (2015).

[48] H. Griesshammer, J. McGovern, and D. R. Phillips, Eur. Phys. J. A 52, 139 (2016).

[49] B. Tiburzi and S. Vayl, Phys. Rev. D 87, 054507 (2013).

[50] B. Tiburzi, Phys. Rev. D 89, 074019 (2014).

[51] A. Deshmukh and B. Tiburzi, Phys. Rev. D 97, 014006 (2018).

[52] J.-W. Lee and B. Tiburzi, Phys. Rev. D 89, 054017 (2014).

[53] J.-W. Lee and B. Tiburzi, Phys. Rev. D 90, 074036 (2014).

[54] L. Foldy, Phys. Rev. Lett. 3, 105 (1959).

[55] M. Bawin and S. A. Coon, Phys. Rev. C 55, 419 (1997).

[56] M. Pitschmann and A. Ivanov, arXiv:1205.5501.

[57] P. Strocchi, Nuovo Cimento 37, 1079 (1965).

[58] G. Shishkin and V. Villalba, J. Math. Phys. (N.Y.) 34, 5037 (1993).

[59] L. Foldy and S. Wouthuysen, Phys. Rev. 78, 29 (1950).

[60] M. Bawin and S. A. Coon, Few-Body Syst. Suppl. 10, 143 (1999).

[61] A. Silenko, J. Math. Phys. (N.Y.) 44, 2952 (2003). 